\section{Efecto de la simvastatina en la concentración de las proteínas de fase aguda después de la cirugía cardíaca}

\author{
Eduardo Tamayo ${ }^{a}$, Óscar Alonso ${ }^{a}$, Francisco Javier Álvarez ${ }^{\mathrm{b}}$, \\ Javier Castrodezac ${ }^{c}$, Santiago Flórez ${ }^{\mathrm{d}}$ y Salvatore di Stefano ${ }^{\mathrm{d}}$
}

a Servicio de Anestesiología y Reanimación. Hospital Clínico Universitario. Valladolid. 'Departamento de Farmacología y Terapéutica. 'Departamento de Medicina Preventiva y Salud Pública. Facultad de Medicina. Universidad de Valladolid. Valladolid.

- Servicio de Cirugía Cardíaca. ICICOR. Hospital Clínico Universitario. Valladolid. España.

\begin{abstract}
FUNDAMENTO Y OBJETIVO: Existe información contradictoria referente a que los efectos pleiotrópicos de dictoria referente a que los efectos pleiotropicos de tervenciones con circulación extracorpórea, ya que tervenciones con circulación extracorpórea, ya que nas de fase aguda.

PACIENTES Y MÉTODO: Se ha realizado un estudio prospectivo y aleatorizado que incluyó a 44 pacientes a los que se efectuó derivación aortocoronaria con circulación extracorpórea. Se dividieron en 2 grupos: $A(n=22)$, formado por pacientes que tomaron simvastatina, y $B(n=22)$, que fue que tomaron simvastatina, y $B(n=22)$, que fue
el grupo control. Se determinaron las concentraciones plasmáticas de proteínas de fase aguda (interleucina 6, fracción $\mathrm{C} 4$ del complemento y proteína $\mathrm{C}$ reactiva).

RESULTADOS: No se observaron diferencias significativas entre ambos grupos en las concentraciones de proteínas de fase aguda ni en las complicaciones postoperatorias. En ambos grupos, las concentrapostoperatorias. En ambos grupos, las concentraciones máximas de interleucina 6 se observaron a
las $6 \mathrm{~h}$ de la cirugía y las de proteína $C$ reactiva a las $48 \mathrm{~h}$. Las concentraciones de $\mathrm{C} 4$ descendieron al inicio de la derivación cardiopulmonar y volvieron a la normalidad a las $48 \mathrm{~h}$.

CONCLUSIONES: La administración de simvastatina a pacientes intervenidos de revascularización miocárdica con circulación extracorpórea no modifica cardica con circulación extracorporea no modifica fase aguda.
\end{abstract}

Palabras clave: Cirugía cardíaca. Derivación cardiopulmonar. Simvastatina. Estatinas. Citocinas. Proteínas de fase aguda. Síndrome de respuesta inflamatoria sistémica.

Effects of simvastatin on acute-phase protein levels after cardiac surgery

BACKGROUND AND OBJECTIVE: There is contradictory evidence as to whether the pleiotropic effects of statins improve morbidity/mortality rates in coronary artery bypass grafting with extracorporeal circulation, as they reduce the protein plasma levels in the acute phase.

PATIENTS AND METHOD: This randomized prospective study included 44 patients undergoing elective coronary artery bypass grafting with extracorporeal coronary artery bypass grafting with extracorporeal group $A(n=22)$, patients taking simvastatin, and group $B$, control $(n=22)$. The plasma levels of in terleukin-6, complement 4 and C-reactive protein were determined.

RESULTS: No significant differences were noted between the 2 groups with respect to the acutephase protein levels, or the postoperative complications. In both groups, compared with the initial levels, interleukin- 6 levels peaked at $6 \mathrm{~h}$ after surlevels, interleukin- 6 levels peaked at $6 \mathrm{~h}$ after sur-
gery and C-reactive protein at $48 \mathrm{~h}$. Complement 4 levels decreased from the start of the cardiopulmonary bypass and returned progressively toward the baseline value at $\mathbf{4 8} \mathrm{h}$ after surgery.

CONCLUSIONS: Simvastatin in patients undergoing coronary artery bypass grafting with cardiopulmonary bypass produces no significant differences in nary bypass produces no significant

Key words: Cardiac surgery. Cardiopulmonary

bypass. Simvastatin. Statin. Cytokines. Acute-phase protein. Systemic inflammatory response syndrome.
En las intervenciones de cirugía cardíaca, con el inicio de la derivación cardiopulmonar se desencadena una intensa respuesta inflamatoria que se caracteriza por la activación del complemento, neutrófilos, endotoxina, elastasa y citocinas proinflamatorias ${ }^{1}$. La interleucina 6 (IL-6) es responsable de la coordinación de la respuesta de fase aguda, que consiste en fiebre, taquicardia, leucocitosis, alteración de la permeabilidad vascular e incremento de la producción de proteínas de fase aguda, como el complemento y la proteína C reactiva (PCR). Las concentraciones plasmáticas de las proteínas de fase aguda se relacionan con la intensidad de la respuesta inflamatoria y con el desarrollo de complicaciones postoperatorias (inestabilidad hemodinámica, fiebre, hemorragia, disfunción multiorgánica, etc.) $)^{2}$.

Los inhibidores de la 3-hidroxi-3-metilglutaril coenzima A reductasa o estatinas son fármacos conocidos por reducir la concentración de colesterol y por sus efectos característicos llamados pleiotrópicos, que consisten en mejora de la disfunción endotelial, incremento de la biodisponibilidad del óxido nítrico y propiedades antioxi-dantes, antiinflamatorias, inmunomoduladoras, antitrombóticas y antiapoptóticas ${ }^{3,4}$. Se ha atribuido a estas propiedades la mejora de los resultados clínicos de los pacientes que presentan síndrome coronario agudo, así como la reducción del riesgo de efectos adversos cardiovasculares y de muerte en diferentes enfermedades cardiovasculares $^{3,4}$, derivadas de la ateromatosis, que se acompañan de fenómenos inflamatorios crónicos en el endotelio vascular. Recientemente se ha apuntado que las estatinas también pueden reducir el riesgo de resultados adversos después de pro- cedimientos cardiovasculares invasivos que se acompañan de respuesta inflamatoria sistémica aguda, como la angioplastia coronaria, la endarterectomía carotídea, la cirugía vascular o la cardíaca ${ }^{3-5}$, si bien es contradictoria la información ${ }^{5,6}$ sobre los efectos de las estatinas en los pacientes a quienes se practica derivación cardiopulmonar.

El objetivo del presente estudio ha sido comprobar si el uso de simvastatina, a la dosis de $20 \mathrm{mg} /$ día por vía oral, que es la más frecuentemente prescrita en la práctica clínica para el tratamiento de la hipercolesterolemia, produce una reducción de las proteínas de fase aguda (IL-6, PCR y complemento) liberadas durante el estado inflamatorio agudo que sigue a la derivación cardiopulmonar.

\section{Pacientes y método}

Se diseñó un estudio prospectivo y aleatorizado en el que se incluyó a 44 pacientes sometidos a intervenciones de cirugía electiva de derivación aortocoronaria. Desde mayo de 2006 hasta abril de 2007 se evaluó a los pacientes programados para cirugía electiva de revascularización miocárdica con circulación extracorpórea para su posible inclusión en el estudio. Se excluyó a aquellos con insuficiencia renal, insuficiencia hepática, insuficiencia cardíaca congestiva, fracción de eyección menor del 40\%, enfermedades inflamatorias o inmunitarias, infarto de miocardio en los 6 meses previos, uso preoperatorio de corticoides y tratamiento con fármacos hipolipemiantes durante las últimas 3 semanas. En todos los casos, antes de la intervención quirúrgica se retiraron los medicamentos antiagregantes. Se dividió a los pacientes en 2 grupos: grupo $A(n=22)$, que tomó $20 \mathrm{mg} /$ día de simvastatina durante 3 semanas hasta el día de la cirugía, y grupo $B$ o control $(n=22)$, que no recibió dicho medicamento. Excepto el perfusionista, ningún miembro del equipo médico conoció la aleatorización. El protocolo de estudio fue aprobado por la Comisión de Investigación del hospital y los pacientes dieron el consentimiento por escrito.

En todos los pacientes se utilizaron procedimientos estándar. En la inducción y el mantenimiento de la anestesia se utilizaron fentanilo, midazolam, etomidato, sevoflurano y rocuronio a dosis según el peso cor-

Correspondencia: Dr. E. Tamayo.

Servicio de Anestesiología y Reanimación. Hospital Clínico Universitario.

Avda. Ramón y Cajal, 3. 47005 Valladolid. España.

Correo electrónico: tamayo@med.uva.es 


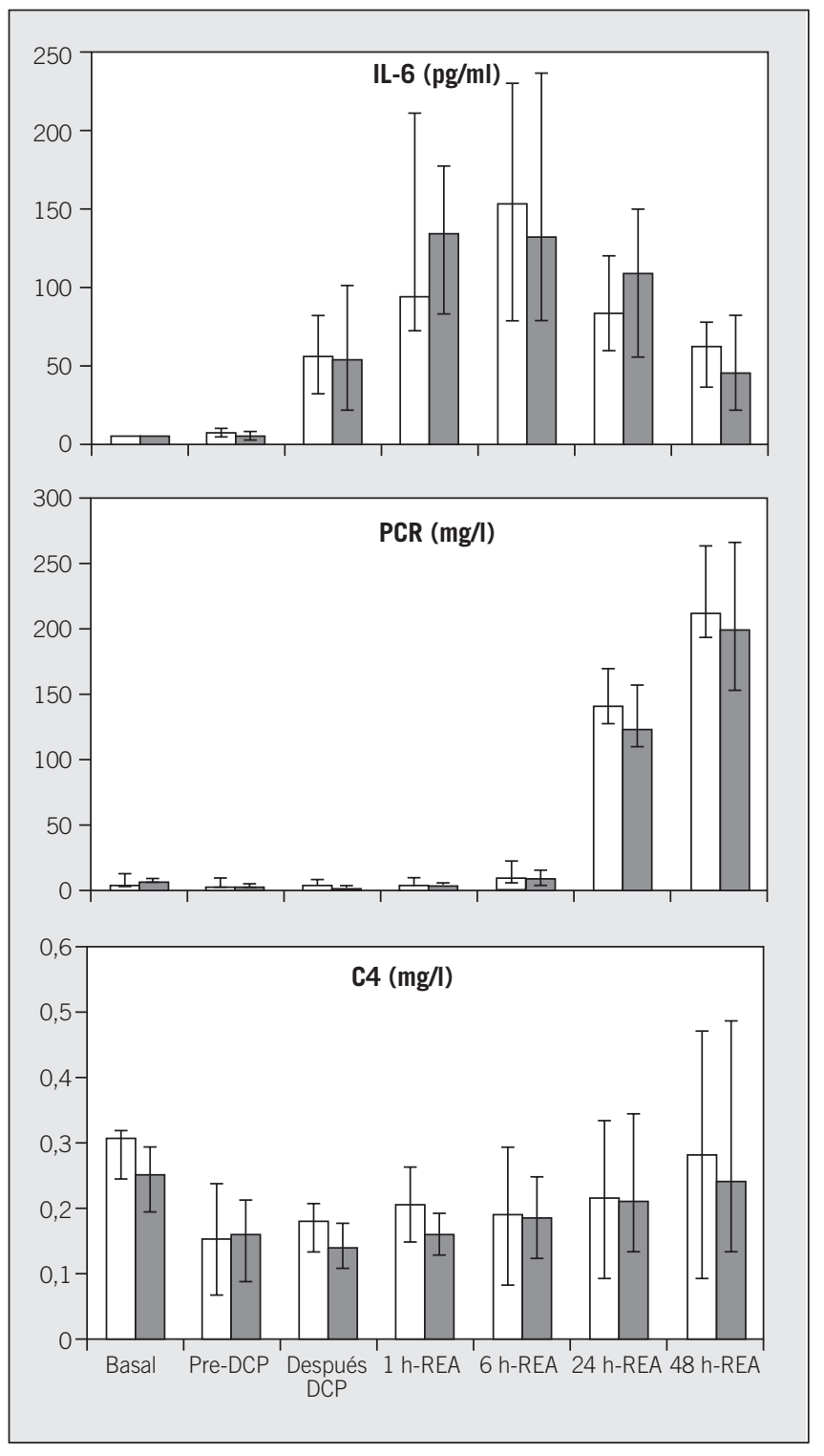

gráficas, clínicas y datos operatorios (tabla 1). No se observaron diferencias estadísticamente significativas $(p>0,05)$ entre ambos en las mediciones hemodinámicas y en las variables postoperatorias (tabla 1 ).

No hubo diferencias significativas entre los 2 grupos en los valores plasmáticos de IL-6, PCR y C4 en ningún momento del estudio. En ambos grupos el valor máximo de IL-6, comparado con los valores basales, se observó a las $6 \mathrm{~h}$ de la cirugía ( $p<0,0001)$. Las concentraciones de PCR se incrementaron a partir de las $6 \mathrm{~h}$ de ingresar en la Unidad de Reanimación y alcanzaron el pico a las 48 h ( $p$ $<0,0001)$. Las concentraciones de C4 descendieron ( $p<0,0001$ ) al inicio de la derivación cardiopulmonar y se incrementaron progresivamente, para volver a la normalidad a las $48 \mathrm{~h}$ de la cirugía ( $p$ $>0,05)$.

\section{Discusión}

Fig. 1. Perfil de cambios temporales observados en las concentraciones de interleucina 6 (IL-6), protelna $C$ reactiva (PCR) y fracción C4 del complemento en pacientes que tomaron simvastatina (barra oscura) y en el grupo control (barra blanca). Los datos se presentan como mediana e intervalos intercuartílicos. Basal: antes de la intervención; preDCP: antes de la derivación cardiopulmonar; des pués DCP: después de la derivación cardiopulmo nar: 1 h-REA: al ingresar en la Unidad de Reanimación; 6 h-REA: $6 \mathrm{~h}$ des pués de la cirugía; 24 hREA: 24 h después de la cirugía; 48 h-REA: 48 después de la cirugía.

poral. Para la circulación extracorpórea se empleó oxigenador de membrana (D903 Avant, Dideco, Mirandola, Italia). El circuito extracorpóreo se purgó con $1.500 \mathrm{ml}$ de Ringer lactato más $200 \mathrm{ml}$ de manitol al $20 \%$. Se mantuvo a los pacientes en moderada hipotermia ( $32{ }^{\circ} \mathrm{C}$ de temperatura nasofaríngea). Después de pinzar la aorta, como protección miocárdica se administró cardioplejía hemática.

Las muestras sanguíneas para las determinaciones de la fracción C4 del complemento (C4), PCR e IL-6 se extrajeron de la arteria radial en distintos momentos del estudio (fig. 1). Se introdujeron en tubos con ácido edético, se centrifugaron inmediatamente a $4{ }^{\circ} \mathrm{C}$ y se guardaron a $-80{ }^{\circ} \mathrm{C}$ hasta su análisis. La IL-6 se determinó mediante enzimoinmunoanálisis (CLB, Department of Immune Reagents, Amsterdam, Países Bajos). Las concentraciones no se corrigieron según la dilución de la extracorpórea. Las concentraciones de C4, PCR y albúmina se determinaron mediante nefelometría (Behring Diagnostics Benelux NV, Behring Nephelometer Analyzer, Behringwerke AG, Marburg, Alemania), según normas de la casa comercial

Se registraron datos demográficos y clínicos de los pacientes, variables intraoperatorias y de evolución postoperatoria (tabla 1), además de las constantes hemodinámicas (presión arterial media, presión venosa central y frecuencia cardíaca).

\section{Análisis estadístico}

El número de pacientes necesarios para cada grupo se calculó basándose en estudios previos sobre los valores de IL-6 durante la cirugía cardíaca con derivación cardiopulmonar ${ }^{1,2}$. Para demostrar nuestra hipótesis, esto es, que el tratamiento con simvastatina reduce la concentración de IL-6 un 30\%, necesitaríamos una población de 20 pacientes en cada grupo para detectar esta diferencia con un error alfa de 0,5 y una potencia de 0,80

Los datos se presentan como media (desviación estándar). Los valores de IL-6, PCR y C4 se presentan como mediana e intervalo intercuartílico. Para el análisis estadístico de los datos se utilizó el programa SPSS, versión 14, y se aplicaron las pruebas estadísticas de la $x^{2}$, de la $t$ de Student y el test exacto de Fisher. Para analizar las diferencias con respecto al momento basal y entre grupos, se realizó un análisis de la variancia para medidas repetidas de 2 colas (ANOVA, seguido del test de Scheffe). Se consideraron estadísticamente significativos los valores de p iguales o menores de 0,05.

\section{Resultados}

Los 2 grupos fueron homogéneos y comparables en sus características demo-
En este estudio prospectivo y aleatorizado, que evalúa el efecto de las estatinas sobre el modelo de respuesta inflamatoria sistémica aguda que provoca la circulación extracorpórea durante las intervenciones de revascularización miocárdica en pacientes con cardiopatía isquémica estable, no se han observado diferencias significativas en las concentraciones plasmáticas de las proteínas de fase aguda (IL-6, PCR, C4) ni en la evolución postoperatoria entre los pacientes que tomaron o no simvastatina a dosis de $20 \mathrm{mg} /$ 24 h por vía oral.

Estudios previos ${ }^{3,4}$ han evidenciado que el tratamiento preoperatorio con estatinas se asocia a una reducción de la mortalidad en pacientes operados de endarterectomía carotídea, cirugía vascular mayor o angioplastia coronaria. Sin embargo, sus resultados en cirugía cardíaca son contradictorios ${ }^{5,6}$. En un estudio prospectivo de cohortes $^{5}$ en 5.436 pacientes con derivación aortocoronaria, el uso preoperatorio de estatinas se asoció a un descenso de mortalidad, morbilidad y estancia hospitalaria. Se ha hipotetizado que estos efectos beneficiosos son debidos a sus propiedades pleiotrópicas. Sin embargo, otros autores ${ }^{6}$, en un estudio realizado en 5.469 pacientes operados de derivación aortocoronaria, no han evidenciado que la administración de estatinas influya en la morbilidad ni en la mortalidad después de la cirugía cardíaca, hecho que explican porque la magnitud de la respuesta inflamatoria desencadenada por la derivación cardiopulmonar durante la cirugía cardíaca es mayor que el impacto que pudiera tener el uso de estatinas para su prevención.

Así, se ha observado que la administración de 20 mg/día de atorvastatina du- 
TABLA 1

\section{Características demográficas y clínicas, datos operatorios y evolución postoperatoria}

\begin{tabular}{|c|c|c|c|}
\hline Variable & Simvastatina $(n=22)$ & Control $(n=22)$ & $\mathrm{p}$ \\
\hline Edad media (años) & $67,7(7,3)$ & $68,0(6,9)$ & 0,656 \\
\hline Varones & $17(77,3 \%)$ & $18(81,8 \%)$ & 0,709 \\
\hline Fumadores & $5(22,7 \%)$ & $10(45,5 \%)$ & 0,112 \\
\hline Diabetes & $6(27,3 \%)$ & $9(40,9 \%)$ & 0,340 \\
\hline Hipertensión arterial & $5(22,7 \%)$ & $11(50,0 \%)$ & 0,060 \\
\hline Clase funcional (NYHA) & $2,09(0,29)$ & $2,05(0,39)$ & 0,787 \\
\hline Nitritos & $15(68,2 \%)$ & $16(72,7 \%)$ & 0,741 \\
\hline Bloqueadores beta & $6(27,3 \%)$ & $10(45,5 \%)$ & 0,210 \\
\hline Antagonistas del calcio & $4(18,2 \%)$ & $7(31,8 \%)$ & 0,296 \\
\hline IECA & $5(22,7 \%)$ & $9(40,9 \%)$ & 0,195 \\
\hline ARA-II & $2(9,1 \%)$ & $3(13,6 \%)$ & 0,163 \\
\hline Fracción de eyección & $61,2(11,4 \%)$ & $63,3(10,3 \%)$ & 0,672 \\
\hline Creatinina preoperatoria (mg/dl) & $1(0,5)$ & $1(0,5)$ & 0,631 \\
\hline Número de puentes coronarios & $3,4(0,8)$ & $3,0(0,8)$ & 0,127 \\
\hline Tiempo de circulación extracorpórea (min.) & $106,8(26,9)$ & $96,2(24,6)$ & 0,853 \\
\hline Tiempo de pinzamiento de la aorta (min.) & $70,0(23,9)$ & $62,7(15,5)$ & 0,207 \\
\hline Defunciones & 0 & 0 & \\
\hline Creatinina postoperatoria $(\mathrm{mg} / \mathrm{dl})$ & $1,2(0,5)$ & $1,3(0,8)$ & 0,561 \\
\hline $\mathrm{pO}_{2} / \mathrm{FiO}_{2}$ llegada-REA & $300,9(95,8)$ & $246,6(60,4)$ & 0,103 \\
\hline $\mathrm{pO}_{2} / \mathrm{FiO}_{2} 6 \mathrm{~h}-\mathrm{REA}$ & $264,6(73,2)$ & $243,5(63,3)$ & 0,952 \\
\hline $\mathrm{pO}_{2}^{2} / \mathrm{FiO}_{2} 24$ h-REA & $236(44,8)$ & $246,7(65,7)$ & 0,604 \\
\hline $\mathrm{pO}_{2} / \mathrm{FiO}_{2} 48 \mathrm{~h}-\mathrm{REA}$ & $208,7(42,4)$ & $211,9(38,0)$ & 0,410 \\
\hline Hemorragia (ml/24 h) & $955,8(478,8)$ & $1.031(537,7)$ & 0,810 \\
\hline Tiempo de ventilación (h) & $10,7(6,8)$ & $16,4(23,9)$ & 0,038 \\
\hline Fibrilación auricular & 0 & $1(4,5 \%)$ & 0,312 \\
\hline Creatincinasa $(\mathrm{U} / \mathrm{l})$ & $799,1(842,8)$ & $601,5(358,1)$ & 0,278 \\
\hline CK-MB (U/I) & $92,9(123,4)$ & $99,0(216,1)$ & 0,491 \\
\hline Transfusión de hemoderivados & $17(77,3 \%)$ & $18(81,8 \%)$ & 0,140 \\
\hline Inotrópicos & $13(59,9 \%)$ & $9(40,9 \%)$ & 0,834 \\
\hline Estancia media en REA (días) & $2,5(2,3)$ & $2,4(1,8)$ & 0,317 \\
\hline
\end{tabular}

Valores expresados como número de pacientes (porcentaje) o media (desviación estándar).

ARA-II: antagonistas de los receptores de la angiotensina II; CK-MB: isoenzima MB de la creatincinasa; $\mathrm{FiO}_{2}$ : fracción inspiratoria de oxígeno; IECA: inhibidores de la enzima de conversión de angiotensina; NYHA: New York Heart Association; $\mathrm{pO}_{2}$ : presión parcial de oxígeno; REA: Unidad de Reanimación; 6 h-REA: 6 h después de la cirugía; 24 h-REA: 24 h después de la cirugía; 48 h-REA: 48 h después de la cirugía.

rante 3 semanas antes de la cirugía reduce las concentraciones de IL-6 ${ }^{7}$. Liakopoulos et $a^{8}{ }^{8}$, en un estudio realizado en 18 pacientes que tomaron diferentes estatinas (atorvastatina, pravastatina y simvastatina) a dosis diarias distintas (10; 20, y $40 \mathrm{mg}$ ), observaron una atenuación de la liberación de citocinas proinflamatorias (IL-6) y antiinflamatorias (IL-10) después de la cirugía cardíaca con circulación extracorpórea. Sin embargo, en otro estudio ${ }^{9}$ en el que se administraban $40 \mathrm{mg}$ de atorvastatina la noche anterior y la mañana previa a la cirugía no se logró demostrar ninguna modificación en las concentraciones de IL-6. Coincidiendo con la opinión de Ali y Buth ${ }^{6}$, creemos que en nuestro estudio la ausencia de cambios en las concentraciones de proteínas de fase aguda de los pacientes tratados con simvastatina se explicaría porque el estímulo más importante para su aumento es la agresión quirúrgica y los sistemas extracorpóreos ${ }^{10}$. Por consiguiente, a la hora de interpretar estos resultados contradictorios entre estudios hay que tener en cuenta que las condiciones clínicas de cada estudio son dife- rentes (dosis de estatinas, tipo de estatina, tipo de circuito extracorpóreo, uso de aprotinina, tipo de cardioplejía, etc.).

En nuestro estudio, en ambos grupos el patrón de cambios observados en los valores de IL-6, PCR y C4 es coincidente con los datos de la literatura médica ${ }^{1,2}$ Durante la derivación cardiopulmonar se produce una activación del complemento, principalmente a través de la vía alternativa, que es inducido mediante el contacto de la sangre con la superficie del circuito extracorpóreo. Después de la administración de protamina y de la subsiguiente formación del complejo heparinaprotamina, se activa el complemento a través de la vía clásica ${ }^{2}$. Las concentraciones plasmáticas de C4 representan la activación de la vía clásica del complemento, y en nuestro estudio estas concentraciones descendieron al principio como consecuencia de la hemodilución de la derivación cardiopulmonar, para recuperar a las 48 h sus valores basales. Esto se explica porque la PCR, prototipo de proteína de fase aguda humana, activa el complemento a través de la vía clásica y sus concentraciones no aumenta- ron notablemente hasta las $24 \mathrm{~h}$ del inicio de la cirugía, para culminar al segundo día. Este retraso entre el estímulo quirúrgico y el valor máximo de la PCR coincide con lo comunicado en la literatura médica y se debe a que sus concentraciones no se incrementan hasta que aumentan los valores de IL-6 ${ }^{2}$.

Nuestras conclusiones se ven limitadas porque únicamente evaluamos la dosis de 20 mg/día de simvastatina, administrada durante 3 semanas. Posiblemente dosis mayores (40 y 60 mg/día) puedan tener un efecto diferente.

Podemos concluir que la administración de simvastatina según las condiciones de nuestro estudio no modifica la concentración sanguínea de proteínas de fase aguda (IL-6, PCR, C4).

\section{REFERENCIAS BIBLIOGRÁFICAS}

1. Westaby S. Organ dysfunction after cardiopulmonary bypass. A systemic inflammatory reaction initiated by the extra-corporeal circuit. Intensive Care Med. 1987;13:89-95.

2. Bruins $P$, Te Velthuis $H$, Yazdanbakhsh AP, Jansen PG, Van Hardevelt FW, De Beaumont EM, et al. Activation of the complement system during and after cardiopulmonary bypass surgery: postsurgery activation involves C-reactive protein and is associated with postoperative arrhythmia. Circulation 1997.96.3542-8.

3. Gómez-Fernández P. Estatinas y efectos beneficiosos. Med Clin (Barc). 2005;125:578-9

4. Gómez-Doblas JJ, Jiménez-Navarro MF, GarcíaPinilla JM, Rodríguez-Bailón I, Robledo J, Cabrera $F$, et al. Influencia del tratamiento temprano con estatinas en la función endotelial tras el infarto de miocardio en pacientes con valores normales de colesterol. Estudio VAATOPE (VALue of ATOrvastatin in Postinfarction Endothelium). Med Clin (Barc). 2006;126:325-8

5. Collard CD, Body SC, Shernan SK, Wang S, Mangano DT; Multicenter Study of Perioperative Ischemia (MCSPI) Research Group, Inc; Ischemia Research and Education Foundation (IREF) Investigators. Preoperative statin therapy is associated with reduced cardiac mortality alter coronary artery bypass graft surgery. J Thorac Cardiovasc Surg. 2006;132:392-400

6. Ali IS, Buth KJ. Preoperative statin use and outcome following cardiac surgery. Int J Cardiol. 2005; 103:12-8.

7. Chello M, Patti G, Candura D, Mastrobuoni S, D Sciascio G, Felice F, et al. Effects of atorvastatin on systemic inflammatory response after coronary bypass surgery. Crit Care Med. 2006;34: nary by 7 .

8. Liakopoulos OJ, Dörge H, Schmitto JD, Nagorsnik U, Grabedünkel J, Schoendube FA. Effects of preoperative statin therapy on cytokines after cardiac surgery. Thorac Cardiovasc Surg. 2006; cardiac surge

9. Prondzinsky R, Knupfer A, Loppnow H, Redling F, Lehmann DW, Stabenow I, et al. Surgical trauma affects the proinflammatory status after cardiac surgery to a higher degree than cardiopulmonary bypass. J Thorac Cardiovasc Surg. 2005; 129:760-6.

10. Florens E, Salvi S, Peynet J, Elbim C, Mallat Z, Bel $A$, et al. Can statins reduce the inflammatory response to cardiopulmonary bypass? A clinical study. J Card Surg. 2001;16:232-9. 